\title{
GENERALIZED BONUS-MALUS SYSTEMS WITH A FREQUENCY AND \\ A SEVERITY COMPONENT ON AN INDIVIDUAL BASIS \\ IN AUTOMOBILE INSURANCE*
}

BY

Rahim Mahmoudvand And Hossein Hassani

\begin{abstract}
Frangos and Vrontos (2001) proposed an optimal bonus-malus systems with a frequency and a severity component on an individual basis in automobile insurance. In this paper, we introduce a generalized form of those obtained previously.
\end{abstract}

\section{KEYWORDS}

Bonus-malus systems, frequency component, severity component.

\section{INTRODUCTION}

Dionne and Vanasse $(1989,1992)$ have presented a bonus-malus systems (BMS) that integrates risk classification and experience rating based on the number of claims of each policyholder. This BMS is derived as a function of the years that the policyholder is in the portfolio, of the number of accidents and of the significant - for the number of accidents - individual characteristics.

Frangos and Vrontos (2001) extend BMS model by introducing the severity component. They proposed a BMS that integrates a priori and a posteriori information on an individual basis based on the both frequency and the severity component. This BMS will be derived as a function of the years that the policyholder is in the portfolio, of the number of accidents, of the exact size of loss that each one of these accidents incurred, and of the significant individual characteristics for the number of accidents and for the severity of the accidents. Some of the a priori rating variables that could be used are the age, the sex and the place of residence of the policyholder, the age, the type and the cubic capacity of the car, etc.

* This research was in part supported by a grant (No. 8716) from Statistical Research and Training Center, Iran. 
One of the reasons for the development of a generalized model which integrates a priori and a posteriori information is that premiums should vary simultaneously with the variables that affect the distribution of the number of claims and the size of loss distribution. It is assumed that the number of claims of each policyholder is independent from the severity of each claim in order to deal with the frequency and the severity component separately. In this paper, we present a generalized form of those obtained in Frangos and Vrontos (2001).

\section{Generalized BOnUS-MALUS Systems}

Consider an individual $i$ with an experience of $t$ periods. Assume that the number of claims of the individual $i$ for period $j$, denoted as $K_{i}^{j}$, follows the Poisson distribution with parameter $\lambda_{i}^{j}$, and $K_{i}^{j}$ are independent. The expected number of claims of the individual $i$ for period $j$ is then denoted by $\lambda_{i}^{j}$ and consider that it is a function of the vector of $h$ individual's characteristics, denoted as $\mathbf{c}_{i}^{j}=\left(c_{i, 1}^{j}, \ldots, c_{i, h}^{j}\right)$, which represent different a priori rating variables. Specifically assume that $\lambda_{i}^{j}=\exp \left(\mathbf{c}_{i}^{j} \boldsymbol{\beta}^{j}\right) U_{i}$, where $\boldsymbol{\beta}^{j}$ is the vector of the coefficients. If we assume that the $U_{i}$ are independent and identically distributed over time and $U_{i}$ follows a gamma distribution with parameters $(a, a)$ and p.d.f:

$$
f_{U_{i}}\left(u_{i}\right)=\frac{a^{a}}{\Gamma(a)} u_{i}^{a-1} e^{-a u_{i}} .
$$

Using the Bayes theorem one finds that the posterior structure function for a policyholder with $K_{i}^{1}, \ldots, K_{i}^{t}$ claim history and $\mathbf{c}_{i}^{1}, \ldots, \mathbf{c}_{i}^{t+1}$ characteristics is gamma with updated parameters

$$
\left(a+\sum_{j=1}^{t} K_{i}^{j}, \frac{a+\sum_{j=1}^{t} \exp \left(\mathbf{c}_{i}^{j} \boldsymbol{\beta}^{j}\right)}{\exp \left(\mathbf{c}_{i}^{t+1} \boldsymbol{\beta}^{t+1}\right)}\right),
$$

Let us consider the situation in which the vector of individual characteristics remains the same from one year to the next. If one assumes that $\mathbf{c}_{i}^{1}=\ldots=$ $\mathbf{c}_{i}^{t+1}=\mathbf{c}_{i}$ and $\beta^{1}=\ldots=\beta^{t}=\beta$, then (1) is simplified to

$$
\left(a+\sum_{j=1}^{t} K_{i}^{j}, \frac{a}{\exp \left(\mathbf{c}_{i} \boldsymbol{\beta}\right)}+t\right),
$$

which coincides with those obtained by Frangos and Vrontos (2001). That is, the obtained results here can be considered as a general form of the results obtained previously, and can be used to calculate bonus-malus systems with a 
frequency and a severity component on an individual basis in automobile insurance, in general form.

In summary, if the vector of individual characteristics remains the same from one year to the next, (1) and (2) coincide. For example, if $\mathbf{c}_{i}$ is the sex/ occupation of the driver, the sex and the impact of the sex/occupation on the number of accidents and the severity of each accident does not change over time. In this case the generalized form (1) is reduced to the simple form (2). Otherwise, if individual characteristics do vary substantially from one period to the next, the generalized form (1) should be used. For example, some characteristics such as age of the driver, age of the car, and experience of the driver have significant impact on the distribution of frequency and severity of accidents and change over time.

Note that the updated gamma parameters in (1) include information up to time $t+1$ at least for individual characteristics. That is, the gamma parameters include $\mathbf{c}_{i}^{t+1}$. It should be noted that we have characteristic information for any individuals at time $t+1$ to estimate the parameters.

The insurer needs to calculate the best estimator of the true expected number of accidents at period $t+1$. Let $\hat{\lambda}_{i}^{t+1}\left(K_{i}^{1}, \ldots, K_{i}^{t} ; \mathbf{c}_{i}^{1}, \ldots, \mathbf{c}_{i}^{t+1}\right)$ denote this estimator which is a function of past experience over the $t$ periods $\left(K_{i}^{1}, \ldots, K_{i}^{t}\right)$ and of known characteristics over the $t+1$ periods $\left(\mathbf{c}_{i}^{1}, \ldots, \mathbf{c}_{i}^{t+1}\right)$. Using the classical quadratic loss function one can find that:

$$
\begin{aligned}
& \hat{\lambda}_{i}^{t+1}\left(K_{i}^{1}, \ldots, K_{i}^{t} ; \mathbf{c}_{i}^{1}, \ldots, \mathbf{c}_{i}^{t+1}\right) \\
& =\exp \left[\mathbf{c}_{i}^{t+1} \boldsymbol{\beta}^{t+1}\right]\left(\frac{a+\sum_{j=1}^{t} K_{i}^{j}}{a+\sum_{j=1}^{t} \exp \left(\mathbf{c}_{i}^{j} \boldsymbol{\beta}^{j}\right)}\right),
\end{aligned}
$$

If one substitutes the value of zero for $t\left(\right.$ then $\sum_{j=1}^{t} K_{i}^{j}=0$ and $\left.\sum_{j=1}^{t} \exp \left(\mathbf{c}_{i}^{j} \boldsymbol{\beta}^{j}\right)=0\right)$ into (3), the answer would be $\exp \left(\mathbf{c}_{i}^{1} \boldsymbol{\beta}^{1}\right)$ which implies that only a priori rating is used in the first period.

For severity component,consider an individual $i$ with an experience of $t$ periods. Assume that $X_{i, k}^{j}$ is denoted the loss incurred from his claim $k$ for the period $j$. Then, the information we have for his claim size history will be in the form of a vector $X_{i, 1}^{1}, \ldots, X_{i, K_{i}^{t}}^{t}$, and the total claim amount for the specific policyholder over the $t$ periods that he is in the portfolio will be equal to $\sum_{j=1}^{t} \sum_{k=1}^{K_{i}^{j}} X_{i, k}^{j}$. We assume that $X_{i, k}^{j}$ follows an exponential distribution with parameter $y_{i}^{j}$. The parameter $y_{i}^{j}$ denotes the mean or the expected claim severity of a policyholder $i$ in period $j$. As we have already said, all policyholders do not have the same expected claim severity, their cost for the insurer is different and 
thus it is fair each policyholder to pay a premium proportional to his mean claim severity. Consider that the expected claim severity is a function of the vector of the $h$ individual's characteristics, denoted as $\mathbf{d}_{i}^{j}=\left(d_{i, 1}^{j}, \ldots, d_{i, h}^{j}\right)$, which represent different a priori rating variables. Specifically assume that $y_{i}^{j}=$ $\exp \left(\mathbf{d}_{i}^{j} \gamma^{j}\right) W_{i}$, where $\gamma^{j}$ is the vector of the coefficients. If we assume that the $W_{i}$ are independent and identically distributed over time and $W_{i}$ follows a inverse gamma distribution with parameters $(s, s-1)$ and p.d.f:

$$
f_{W_{i}}\left(w_{i}\right)=\frac{(s-1)^{s}}{\Gamma(s)} w_{i}^{-s-1} \exp \left(-\frac{s-1}{w_{i}}\right) .
$$

Using the Bayes theorem the posterior distribution of the mean claim severity for a policyholder with claim sizes $X_{i, 1}^{1}, \ldots, X_{i, K_{i}^{t}}^{t}$ in $t$ periods and characteristics $\mathbf{d}_{i}^{1}, \ldots, \mathbf{d}_{i}^{t+1}$ is inverse gamma with the following updated parameters:

$$
\left(s+K,\left[(s-1)+\sum_{j=1}^{t} \frac{\sum_{k=1}^{K_{i}^{j}} X_{i, k}^{j}}{\exp \left(\mathbf{d}_{i}^{j} \gamma^{j}\right)}\right] \exp \left(\mathbf{d}_{i}^{t+1} \gamma^{t+1}\right)\right),
$$

where $K$ denotes the total number of claims of policyholder $i$ in $t$ periods. Similar to those discussed above regarding the frequency components, if one assumes that $\mathbf{d}_{i}^{1}=\ldots=\mathbf{d}_{i}^{t+1}=\mathbf{d}_{i}$ and $\gamma^{1}=\ldots=\gamma^{t}=\gamma$, then (4) is simplified to

$$
\left(s+K,(s-1) \exp \left(\mathbf{d}_{i} \gamma\right)+\sum_{j=1}^{t} \sum_{k=1}^{K_{i}^{j}} X_{i, k}^{j}\right),
$$

which coincides with those obtained by Frangos and Vrontos (2001).

The insurer needs to calculate the best estimator of the expected claim severity at period $t+1$ using the information from past experience for the claim severity over $t$ periods and of known individual characteristics over the $t+1$ periods. Let us denote this estimator as $\hat{y}_{i}^{t+1}$. Using the classical quadratic loss function one can find that:

$$
\begin{aligned}
& \hat{y}_{i}^{t+1}\left(X_{i, 1}^{1}, \ldots, X_{i, K_{i}^{t}}^{t} ; \mathbf{d}_{i}^{1}, \ldots, \mathbf{d}_{i}^{t+1}\right) \\
& =\frac{(s-1)+\sum_{j=1}^{t} \frac{\sum_{k=1}^{K_{i}^{j}} X_{i, k}^{j}}{\exp \left(\mathbf{d}_{i}^{j} \gamma^{j}\right)} \exp \left(\mathbf{d}_{i}^{t+1} \gamma^{t+1}\right),}{s+K-1}
\end{aligned}
$$


Now we are able to compute the premiums of the generalized BMS based both on the frequency and the severity component. The premiums of the generalized BMS will be given from the product of the generalized BMS based on the frequency component and of the generalized BMS based on the severity component. Thus it will be

$$
P_{i t+1}=\hat{y}_{i}^{t+1} \times \hat{\lambda}_{i}^{t+1} .
$$

\section{COMPARISON}

Let us now consider the discrepancy between the obtained premiums based on the generalized form presented here, $P_{G}$, and by the simple form of Frangos and Vrontos (2001), $P_{S}$, by means of an example. Here we consider different conditions for 10 individuals, but one can consider more conditions and also more samples. Suppose $t=3, a=1, \sum_{j=1}^{t} K_{i}^{j}=0, s=2, X_{i, k}^{j}=0$ and the historical features for 10 policyholders are those presented in Table 1.

TABLE 1

The OBTAINED PREMIUMS USING PG AND PS.

\begin{tabular}{r|cccc|cccc|cc}
\hline \hline$i$ & $e^{c_{i}^{1} \beta^{1}}$ & $e^{c_{i}^{2} \beta^{2}}$ & $e^{c_{i}^{3} \beta^{3}}$ & $e^{c_{i}^{4} \beta^{4}}$ & $e^{d_{i}^{1} \gamma^{1}}$ & $e^{d_{i}^{2} \gamma^{2}}$ & $e^{d_{i}^{3} \gamma^{3}}$ & $e^{d_{i}^{4} \gamma^{4}}$ & $P G$ & $P S$ \\
\hline 1 & 0.50 & 0.55 & 0.63 & 0.69 & 0.60 & 0.68 & 0.82 & 0.92 & 0.237 & 0.150 \\
2 & 0.80 & 0.83 & 0.85 & 0.86 & 0.72 & 0.68 & 0.65 & 0.59 & 0.149 & 0.162 \\
3 & 1.00 & 1.10 & 1.15 & 1.18 & 0.70 & 0.65 & 0.58 & 0.50 & 0.139 & 0.164 \\
4 & 1.05 & 1.10 & 1.12 & 1.15 & 0.20 & 0.24 & 0.29 & 0.31 & 0.083 & 0.062 \\
5 & 1.13 & 1.10 & 1.08 & 1.05 & 0.70 & 0.67 & 0.62 & 0.60 & 0.146 & 0.170 \\
6 & 0.70 & 0.74 & 0.75 & 0.80 & 0.60 & 0.58 & 0.55 & 0.50 & 0.125 & 0.132 \\
7 & 0.93 & 0.90 & 0.89 & 0.85 & 0.60 & 0.61 & 0.62 & 0.69 & 0.158 & 0.149 \\
8 & 1.20 & 1.18 & 1.12 & 1.10 & 0.30 & 0.27 & 0.23 & 0.20 & 0.049 & 0.069 \\
9 & 0.80 & 0.90 & 1.00 & 1.03 & 1.02 & 0.97 & 0.87 & 0.80 & 0.223 & 0.232 \\
10 & 0.90 & 0.98 & 1.00 & 1.10 & 0.30 & 0.30 & 0.35 & 0.40 & 0.113 & 0.078 \\
\hline \hline
\end{tabular}

The last two columns of Table 1 represent $P_{G}$ and $P_{S}$, respectively. As it appears from Table 1, the premiums obtained by $P_{S}$ and $P_{G}$, for each policyholder, are different for all cases. The premiums calculated by simple form are sometimes less than the generalized form, for example cases 1, 4, 7 and 10, and greater than in other cases. As we mentioned above, $P_{G}$ is calculated based on a combination of the fixed and variable characteristics over time whilst $P_{S}$ is only obtained from the fixed characteristics. Therefore, whenever we have a combination of the fixed and variable characteristics over time, like what are considered in 
Table 1, the results are completely different. In this case, one should use the generalized form to obtain accurate premiums. Otherwise, the calculated premiums would be bigger or smaller than the correct value.

To acquire a better understanding of $P_{G}$ and $P_{S}$ obtained in Table 1, we examine the results presented in the first row of Table 1. Assume individual characteristics are sex of the driver (S), age of the driver (A), age of the car (AC), and average driving per day ( $\mathrm{T}$ ) and also consider the following models:

$$
\begin{aligned}
\mathrm{I} & =\exp \left\{c_{i}^{j} \beta^{j}\right\}=\exp \left\{\alpha_{S}^{j} S-\alpha_{A}^{j} A+\alpha_{A C}^{j} A C+\alpha_{T}^{j} T\right\} \\
\mathrm{II} & =\exp \left\{d_{i}^{j} \gamma^{j}\right\}=\exp \left\{\theta_{S}^{j} S-\theta_{A}^{j} A+\theta_{A C}^{j} A C+\theta_{T}^{j} T\right\}
\end{aligned}
$$

where $\alpha^{j}$ and $\theta^{j}$ are the parameters of the models I and II, respectively. The value of $S$ is set to zero for women and 1 for men. Note that here we use a combination of fixed, $S$ and $T$, and variable characteristics, $A$ and $A C$. The first row in Table 1 was obtained based on the information for a man with $A=43$, $A C=2$, and $T=2.5$ at $t=1$. The detailed results are presented in Table 2 .

TABLE 2

\begin{tabular}{|c|c|c|c|c|c|c|c|c|c|c|c|c|c|c|}
\hline \multirow{3}{*}{$j$} & \multicolumn{4}{|c|}{ characteristics } & \multicolumn{8}{|c|}{ parameters } & \multirow{3}{*}{ I } & \multirow{3}{*}{ II } \\
\hline & \multicolumn{2}{|c|}{ fixed } & \multicolumn{2}{|c|}{ variable } & \multirow[b]{2}{*}{$\alpha_{S}^{j}$} & \multirow[b]{2}{*}{$\alpha_{A}^{j}$} & \multirow[b]{2}{*}{$\alpha_{A C}^{j}$} & \multirow[b]{2}{*}{$\alpha_{T}^{j}$} & \multirow[b]{2}{*}{$\theta_{S}^{j}$} & \multirow[b]{2}{*}{$\theta_{A}^{j}$} & \multirow[b]{2}{*}{$\theta_{A C}^{j}$} & \multirow[b]{2}{*}{$\theta_{T}^{j}$} & & \\
\hline & $\mathrm{S}$ & $\mathrm{T}$ & A & $\mathrm{AC}$ & & & & & & & & & & \\
\hline 1 & 1 & 2.5 & 43 & 2 & 0.090 & 0.026 & 0.110 & 0.050 & 0.125 & 0.024 & 0.114 & 0.050 & 0.50 & 0.60 \\
\hline 2 & 1 & 2.5 & 44 & 3 & 0.090 & 0.025 & 0.104 & 0.040 & 0.128 & 0.022 & 0.120 & 0.050 & 0.55 & 0.70 \\
\hline 3 & 1 & 2.5 & 45 & 4 & 0.092 & 0.024 & 0.100 & 0.049 & 0.127 & 0.021 & 0.124 & 0.049 & 0.63 & 0.82 \\
\hline 4 & 1 & 2.5 & 46 & 5 & 0.090 & 0.024 & 0.105 & 0.047 & 0.126 & 0.020 & 0.119 & 0.046 & 0.69 & 0.92 \\
\hline
\end{tabular}

The Detailed Results of THE FIRST Row IN TABLE 1.

Next, we consider the situation in which the premiums $P_{G}$ are greater/smaller than $P_{S}$. Let us first assume $K=0$. In this case we have:

$$
\frac{P_{G}}{P_{S}}=\frac{\exp \left\{c_{i}^{t+1} \beta^{t+1}\right\}\left(a+t \exp \left\{c_{i}^{j} \beta^{j}\right\} \exp \left\{d_{i}^{t+1} \gamma^{t+1}\right\}\right)}{\frac{1}{t} \sum_{j=1}^{t} \exp \left\{c_{i}^{j} \beta^{j}\right\}\left(a+\sum_{j=1}^{t} \exp \left\{c_{i}^{j} \beta^{j}\right\}\right) \frac{1}{t} \sum_{j=1}^{t} \exp \left\{d_{i}^{j} \gamma^{j}\right\}} .
$$

Therefore, we get 


$$
\frac{P_{G}}{P_{S}}=\left\{\begin{aligned}
>1 \text { if }\left\{\begin{aligned}
\text { i) } \exp \left\{c_{i}^{t+1} \beta^{t+1}\right\} & >\frac{1}{t} \sum_{j=1}^{t} \exp \left\{c_{i}^{j} \beta^{j}\right\} \\
\text { ii) } \exp \left\{d_{i}^{t+1} \gamma^{t+1}\right\} & >\frac{1}{t} \sum_{j=1}^{t} \exp \left\{d_{i}^{j} \gamma^{j}\right\}
\end{aligned}\right. \\
<1 \text { if }\left\{\begin{aligned}
i) \exp \left\{c_{i}^{t+1} \beta^{t+1}\right\} & <\frac{1}{t} \sum_{j=1}^{t} \exp \left\{c_{i}^{j} \beta^{j}\right\} \\
\text { ii) } \exp \left\{d_{i}^{t+1} \gamma^{t+1}\right\} & <\frac{1}{t} \sum_{j=1}^{t} \exp \left\{d_{i}^{j} \gamma^{j}\right\}
\end{aligned}\right.
\end{aligned}\right.
$$

Recall that $\exp \left\{c_{i}^{j} \beta^{j}\right\}=E\left(K_{i}^{j}\right)$ and $\exp \left\{d_{i}^{j} \gamma^{j}\right\}=E\left(X_{i, k}^{j}\right)$. Therefore, (11) can be simplified to

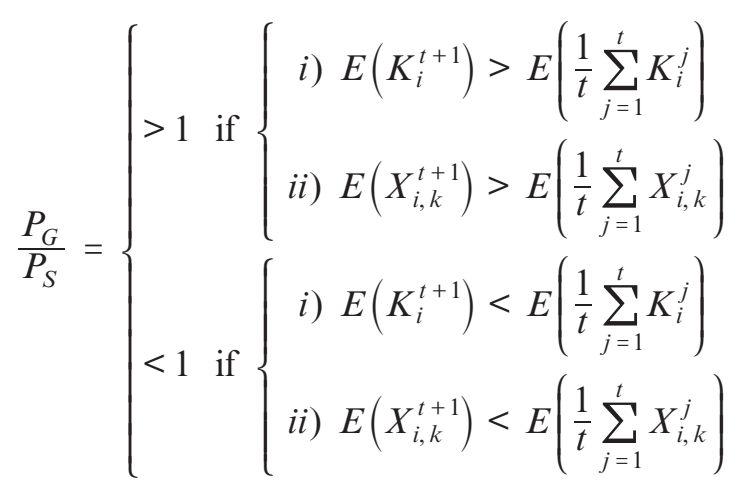

It can be concluded from (12) that $P_{G}<P_{S}$ if severity and frequency components for the period $t+1$ are smaller than the average of $t$ periods. This indication is considerable and directs to obtain optimal BMS. Let us now consider $\frac{P_{G}}{P_{S}}$ for the general case $(K \neq 0)$ :

$$
\frac{\exp \left\{c_{i}^{t+1} \beta^{t+1}+d_{i}^{t+1} \gamma^{t+1}\right\}\left(a+t \exp \left\{c_{i}^{j} \beta^{j}\right\}\right)\left((s-1)+\sum_{j=1}^{t} \frac{\sum_{k=1}^{K_{i}^{j}} X_{i, k}^{j}}{\exp \left\{d_{i}^{j} \gamma^{j}\right\}}\right)}{\frac{1}{t} \sum_{j=1}^{t} \exp \left\{c_{i}^{j} \beta^{j}\right\}\left(a+\sum_{j=1}^{t} \exp \left\{c_{i}^{j} \beta^{j}\right\}\right)\left((s-1) \frac{1}{t} \sum_{j=1}^{t} \exp \left\{d_{i}^{j} \gamma^{j}\right\}+X_{i, .}^{\cdot}\right)},
$$


where $X_{i, .}^{\cdot}=\sum_{j=1}^{t} \sum_{k=1}^{K_{i}^{j}} X_{i, k}^{j}$. Similar to those obtained above, we have the following conditions:

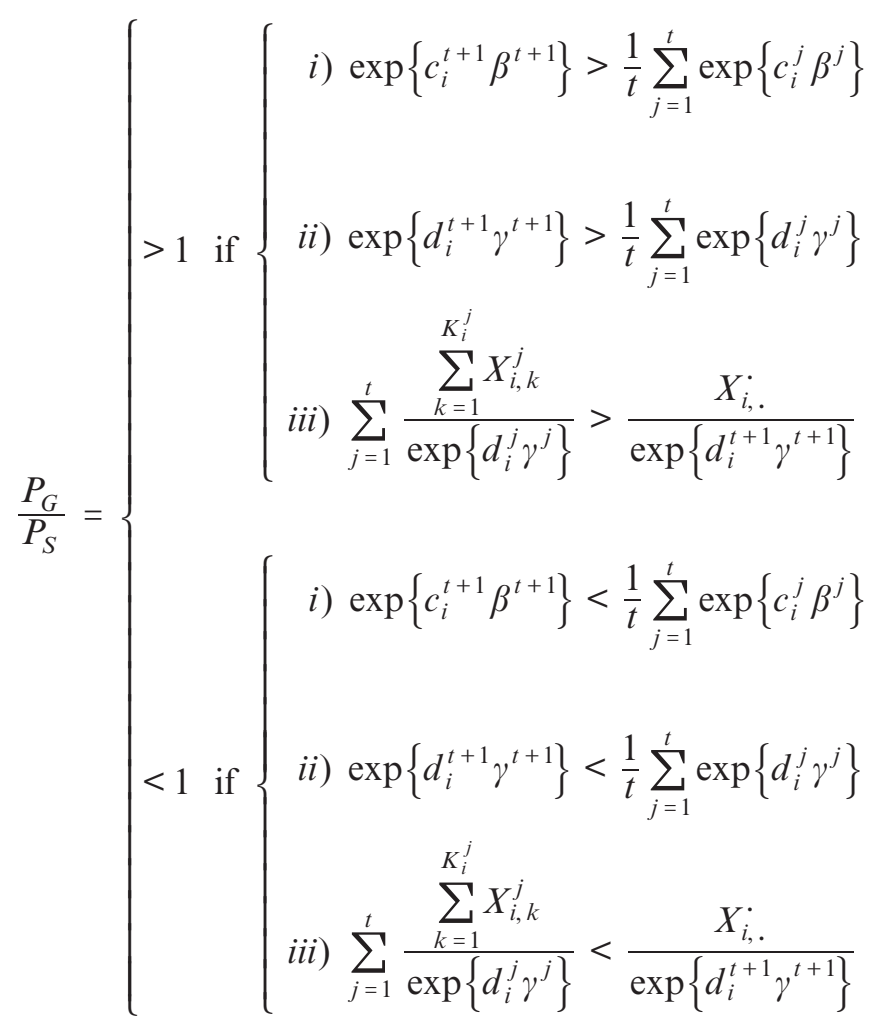

Note that $\frac{\sum_{k=1}^{K_{i}^{j}} X_{i, k}^{j}}{\exp \left\{d_{i}^{j} \gamma^{j}\right\}}$ in (14) is the ratio of the observed losses to the expected losses at period $j$. Thus, if the left hand side of condition iii in (14) is greater that the right hand side, then we conclude that the driver has been faced with more losses than the insurer expected in $t$ periods. Thus, this evidence should be considered in calculating optimal insurance for the next period. Finally, we should mention that the above conditions can be considered as sufficient conditions. However in practice as was shown in Table 1, we have combinations of these conditions.

\section{CONCLUSION}

We introduced a generalized form of optimal BMS with a frequency and a severity component based both on the a priori and the a posteriori classification 
criteria developed previously by Dionne and Vanasse $(1989,1992)$ and Frangos and Vrontos (2001). There are many significant individual characteristics on the distribution of frequency and severity of claims. These characteristics, and their impact, change over time. The generalized BMS obtained here allows us to use both fixed and variable individual characteristics. The results show that one gets much more accurate premiums using the generalized form presented here, if a combination of the fixed and variable characteristics is considered.

\section{ACKNOWLEDGEMENT}

The authors would like to thank the editor Prof. Cairns, Prof. Frangos and Prof. Vrontos for their constructive comments which have led to substantial improvements in this paper.

\section{REFERENCES}

Dionne, G. and Vanasse, C. (1989) A generalization of actuarial automobile insurance rating models: the negative binomial distribution with a regression component, Astin Bulletin 19, 199-212.

Dionne, G. and Vanasse, C. (1992) Automobile insurance ratemaking in the presence of asymmetrical information, Journal of Applied Econometrics 7, 149-165.

Frangos, N.E. and VRONTOS, S.D. (2001) Design of optimal bonus-malus systems with a frequency and a severity component on an individual basis in automobile insurance, Astin Bulletin 33, 1-22.

RAHIM MAHMOUDVAND

Statistical Research and Training Center (SRTC),

Tehran, Iran

and

Group of Statistics,

Payame Noor University of Toyserkan

Toyserkan, Iran.

Hossein HASSANI

Statistical Research and Training Center (SRTC),

Tehran, Iran

and

Centre for Optimisation and Its Applications,

School of Mathematics,

Cardiff University, CF24 4AG, UK

E-Mail: HassaniH@cf.ac.uk 